\title{
Structure and Epigenetic Regulation of Chromatin Fibers
}

\author{
Ping Chen and Guohong Li \\ National Laboratory of Biomacromolecules, CAS Center for Excellence in Biomacromolecules, \\ Institute of Biophysics, Chinese Academy of Sciences, Beijing 100101, China \\ Correspondence: liguohong@ibp.ac.cn
}

\begin{abstract}
In eukaryotes, genomic DNA is hierarchically packaged by histones into chromatin on several levels to fit inside the nucleus. As a central-level structure between nucleosomal arrays and higher-order chromatin organizations, the 30-nm chromatin fiber and its dynamics play a crucial role in gene regulation. However, despite considerable efforts over the past three decades, the fundamental structure and its dynamic regulation of chromatin fibers still remain as a big challenge in molecular biology. Here, we mainly summarize the most recent progress in elucidating the structure of the 30-nm chromatin fiber in vitro and epigenetic regulation of chromatin fibers by chromatin factors, particularly histone variants. In addition, we also discuss recent studies in unraveling the three-dimensional organization of chromatin fibers in situ by genomic approaches and electron microscopy.
\end{abstract}

In eukaryotic cells, the accessibility of DNA is dependent on the packing density of chromatin fibers. Genomic DNA firstly wraps around a histone octamer to form a nucleosome, which is connected by linker DNA to form the primary chromatin structure, the "beads-on-a-string" nucleosomal array. Nucleosomes in the array are further compacted by linker histone H1 to form a 30-nm chromatin fiber - typically regarded as the secondary structure of chromatin. Chromatin fibers are further organized into other higher-levels of chromatin structures, but so far details of these structural levels still remain obscure. The three-dimensional (3D) organization of genomic DNA plays a critical role in regulating DNA-related biological processes, such as gene transcription and DNA replication, repair, and recombination. Elucidating the structure and dynamics of chromatin fibers in molecular details is the key to understanding the epigenetic regulation of gene expression by different chromatin factors.

\section{HIGH-RESOLUTION STRUCTURE OF 30-NM CHROMATIN FIBERS}

It is still a puzzle how genomic DNA is hierarchically organized in eukaryotic cells. From a structural point of view, the DNA double-helix structure discovered by Watson and Crick is surely the most important milestone in molecular biology (Watson and Crick 1953). After more than 40 years, the high-resolution structure of the nucleosome core particle (NCP) has been defined by crystal Xray studies (Luger et al. 1997), which undoubtedly reveals the structural details of histone-histone and histone-DNA interactions within nucleosomes (Fig. 1). Other high-resolution structures of NCPs containing core histones from different species or with a different DNA sequence, different histone variants, or different nucleosome-binding proteins/peptides have been resolved subsequently to in- vestigate the regulation of nucleosome structure as reviewed previously (Cutter and Hayes 2015; McGinty and Tan 2015; Zhu and Li 2016).

Afterward, the manner of how a "beads-on-a-string" nucleosomal array folds into a condensed 30-nm chromatin fiber remains to be determined. Two basic classes of structural models had been proposed previously based on the studies of native 30-nm fibers in nuclei or isolated from nuclei (Finch and Klug 1976; Langmore and Paulson 1983; Widom and Klug 1985; Gerchman and Ramakrishnan 1987; Ghirlando and Felsenfeld 2008). One is the solenoid model, in which nucleosomes are arranged linearly in a one-start solenoid-type helix with bend linker DNA, and the other is the zigzag model, in which nucleosomes zigzag back and forth in a two-start stack of nucleosomes connected by a relatively straight DNA linker (Woodcock et al. 1984; Widom and Klug 1985; Williams et al. 1986). To discriminate between these two structural models, the detailed structure of chromatin fibers needs to be resolved. However, the heterogeneous properties of nucleosomes in native chromatin with different DNA sequences/linker lengths and different histone compositions/modifications make this difficult. The reconstitution of chromatin fibers in vitro using regular tandem repeats of unique nucleosome-positioning DNA sequences and purified histone proteins greatly improves the reproducibility and uniformity for structural analysis (Dorigo et al. 2004). Using this system, Richmond and colleagues first resolved the crystal X-ray structure of chromatin fibers reconstituted by tetranucleosomal arrays with a 20-bp linker DNA at a high concentration of $\mathrm{Mg}^{2+}(120 \mathrm{~mm})$. The structure resolved at $\sim 9 \AA$ resolution reveals two stacks of nucleosomes connected by straight linker DNA, which agrees with the zigzag model (Schalch et al. 2005). Within each nucleosome stack, strong interactions between the H2B-helix $\alpha 1 / \alpha \mathrm{C}$ and the adjacent H2A-helix $\alpha 2$ stabi-

(C) $2017 \mathrm{Chen}$ and $\mathrm{Li}$. This article is distributed under the terms of the Creative Commons Attribution-NonCommercial License, which permits reuse and redistribution, except for commercial purposes, provided that the original author and source are credited. 


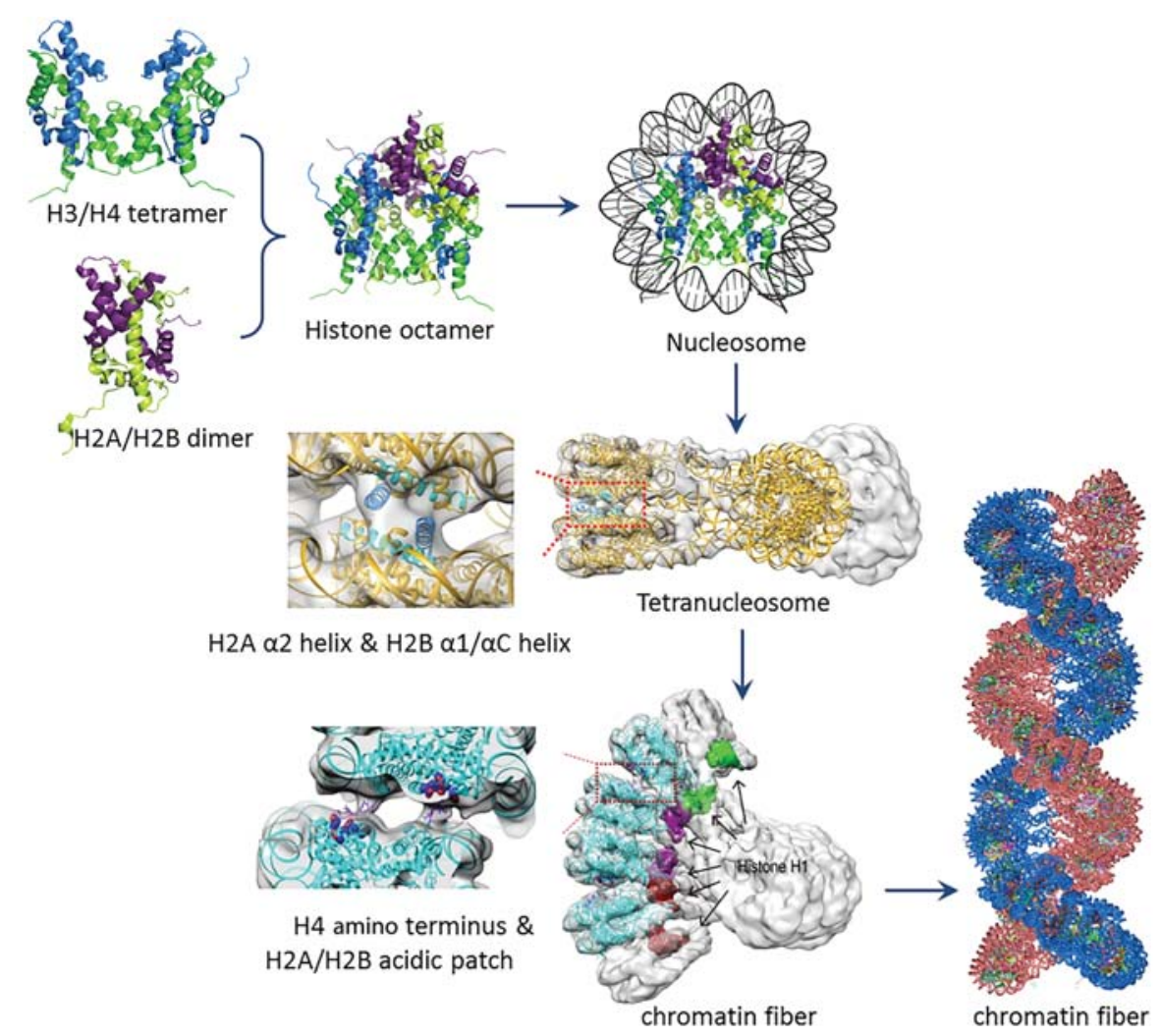

Figure 1. The hierarchical organization of chromatin fibers: 147 base pairs (bp) of DNA wrapped around a histone octamer (with one histone $\mathrm{H} 3 / \mathrm{H} 4$ tetramer and two $\mathrm{H} 2 \mathrm{~A} / \mathrm{H} 2 \mathrm{~B}$ dimers) $~ 1.7$ times in a left-handed manner to form nucleosome, the basic repeating unit of chromatin (Luger et al. 1997). Nucleosomes interact with each other to form the tetranucleosomal structural units, within which the four nucleosomes zigzag back and forth to form two stacks of two nucleosome cores connected by straight linker DNA (Schalch et al. 2005; Song et al. 2014). Strong interactions between the H2B-helix $\alpha 1 / \alpha \mathrm{C}$ and the adjacent $\mathrm{H} 2 \mathrm{~A}-$ helix $\alpha 2$ stabilize each nucleosome stack of tetranucleosomal units. The tetranucleosomal units are twisted against each other in a left-handed manner to form the final doublehelical structure of chromatin fiber (Song et al. 2014). Between the structural units, the H1-H1 interaction and the interactions of H4 amino-terminal tail with the $\mathrm{H} 2 \mathrm{~A} / \mathrm{H} 2 \mathrm{~B}$ acidic patch between the nucleosomal interfaces play central roles.

lize the chromatin fibers (Fig. 1). However, the crystal structure was determined for a tetranucleosomal array, which is too short to form a solenoid structure, and with a very short nucleosome repeat length (NRL; $167 \mathrm{bp})$ in the absence of $\mathrm{H} 1$, which is uncommon in nature.

Recently, we had determined the $3 \mathrm{D}$ cryo-electron microscopy (EM) structures at $\sim 11 \AA$ resolution of longer chromatin fibers reconstituted in vitro from 12-nucleosomal arrays with two different NRLs (177 bp and $187 \mathrm{bp}$ ), which reveal a left-handed double helix twisted with the repeating tetranucleosomal structural units (Song et al. 2014). The structures constitute the largest fragments of chromatin resolved at this high resolution so far and provide new insights into the nucleosome arrangement in helical structure of 30-nm chromatin fibers. The four nucleosomes within the structural unit zigzag back and forth to form two stacks of two nucleosome cores connected by straight linker DNA, which appear very similar to the resolved X-ray structure of a tetranucleosome (Schalch et al. 2005). The tetranucleosomal units are twisted against each other in a left-hand manner to form the final helical structure of 30-nm fibers. The H1-H1 interaction and the interactions of the $\mathrm{H} 4$ amino-terminal tail with the $\mathrm{H} 2 \mathrm{~A}$ / $\mathrm{H} 2 \mathrm{~B}$ acidic patch between the neighboring nucleosomal interfaces play central roles in interaction and twisting between the structural units (Fig. 1). In comparison to the closely stacked nucleosomes within the tetranucleosomal structural unit, the apparently formed gaps between the structural units may provide a platform for histone modifications or other architectural proteins to modulate the internucleosomal surface interactions in the regulation of higher-order chromatin structures.

The linker histone has been considered to play an essential role in the formation of compact chromatin fiber (Thoma et al. 1979; Allan et al. 1980; Bates and Thomas 1981; Thomas 1999). But the molecular details of how linker histones bind to nucleosome and compact chromatin fibers remain to be determined. Our cryo-EM structures, for the first time, clearly address the location and the role of H1 in the formation of the 30-nm chromatin fiber (Song et al. 2014). H1 directly interacts with both the dyad and the entry/exit nucleosomal DNA in a three-contact mode. The binding of H1, which locks the outer DNA wraps of nucleosomes at the enter/exit point as previously discussed (van Holde and Zlatanova 1996; Syed et al. 2010), enhances the stability of the outer nucleosomal wrap with the increase of $\sim 20 \mathrm{~kJ} / \mathrm{mol}$ free energy and accelerates the folding/unfolding rate of the outer wrap 
(Li et al. 2016). In addition, the H1 locates asymmetrically in each nucleosome core with its bulk of globular domain pointed outside the structural units, which allows the selfassociation of $\mathrm{H} 1$ by its globular domain between tetranucleosomal units and imparts an additional twist between each structural unit. However, the $11 \AA$ resolution of the resolved cryo-EM structures cannot give the structural details of $\mathrm{H} 1$ at the atomic levels. Higher resolution structures of chromatin fibers still need to be determined to solve these problems.

\section{SINGLE-MOLECULE STUDY ON THE DYNAMICS OF CHROMATIN FIBERS}

The 30-nm chromatin fiber has been shown to be the first level of the transcriptionally dormant platform (Li et al. 2010). Thus, the structural transition between the $30-\mathrm{nm}$ chromatin fiber and the nucleosomal array plays a critical role in regulating the accessibility of the DNA template ( $\mathrm{Li}$ et al. 2010; Li and Reinberg 2011). Static conformations obtained by our cryo-EM structures of $30-\mathrm{nm}$ chromatin fibers in vitro cannot provide much detailed information for such a dynamic process. In addition, because of the highly dynamic and heterogeneous properties of the chromatin fiber, it is technically challenging to study the dynamics of the chromatin fibers. Recently, several singlemolecule techniques have been applied to investigate the dynamics of nucleosome/chromatin structures (Cui and Bustamante 2000; Pope et al. 2005; Kruithof et al. 2009). Mechanical manipulations of single mononucleosome show that a nucleosome in the absence of linker histone unravels in two major stages: the outer nucleosomal DNA unwrapped at $\sim 3 \mathrm{pN}$ and the inner turn unwrapped at $\sim 10 \mathrm{pN}$ (Brower-Toland et al. 2002; Hall et al. 2009; Bintu et al. 2012). For the mechanical decompaction of higher-order chromatin fibers, Bustamante and colleagues observed a distinct structural transition between "compacted" and "extended" states for the isolated native chicken erythrocyte chromatin fibers at medium ionic strengths $(>40 \mathrm{~mm} \mathrm{NaCl})$ at 5-6 pN, which was attributed to the disruption of internucleosomal interactions for stabilizing the higher-order structures of chromatin fibers (Cui and Bustamante 2000). Van Noort and colleagues used magnetic tweezers to investigate the mechanical stretching of single reconstituted chromatin fibers and also observed the dynamic unfolding behavior of chromatin fibers at low forces $(<6 \mathrm{pN})$ (Kruithof et al. 2009; Meng et al. 2015). They found that the chromatin fibers with 197bp NRL stretch like a Hookian spring, which supports a solenoid topology with a high nucleosome-nucleosome stacking energy. Using magnetic tweezers (Fig. 2A), we investigated the hierarchical organization and dynamics of 30 -nm chromatin fibers reconstituted in vitro in the presence of H1, whose high-resolution 3D cryo-EM structures
A

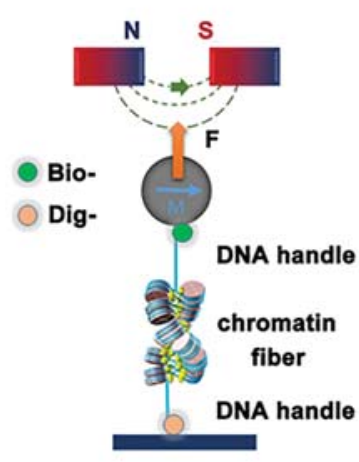

B

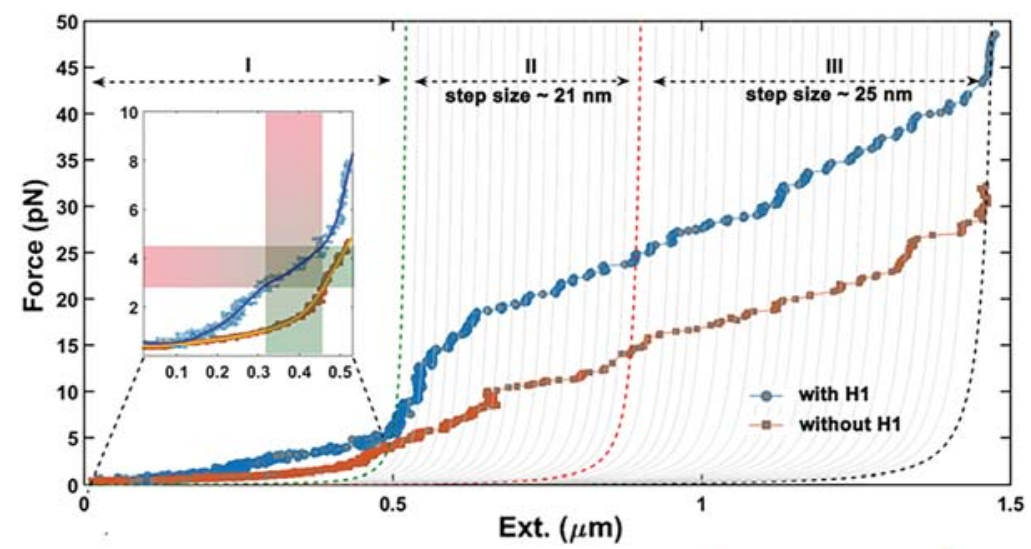

C

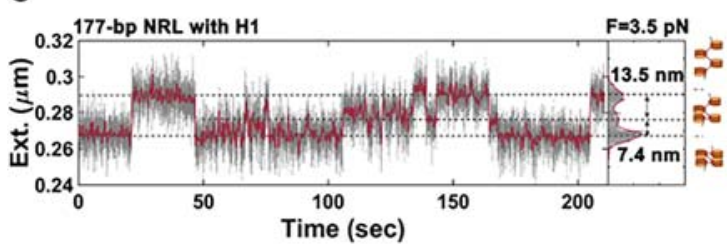

D

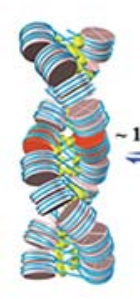

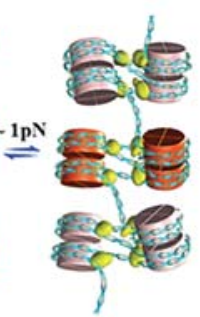

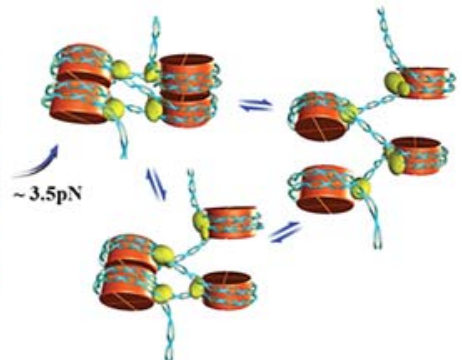

Figure 2. Mechanical unfolding of 30-nm chromatin fibers by single-molecular magnetic tweezers. $(A)$ Schematic setup of the magnetic tweezers used in chromatin fiber studies (not to scale). $(B)$ Comparison of two typical force-extension curves of a chromatin fiber with H1 (blue curve) and without H1 (orange curve) in HE buffer. Three major distinct stages as labeled can be recognized in the forceextension curve of the chromatin fiber with H1. The inset shows the details of Stage I at low forces $(<8 \mathrm{pN})$. (C) Stepwise folding/ unfolding dynamics of tetranucleosomal units with two alternative pathways at $3.5 \mathrm{pN}$ for the 177-bp nucleosome repeat length (NRL)'s chromatin fiber with H1. $(D)$ Model for the dynamic organization of chromatin fibers. The left-handed double-helical chromatin fiber unfolds to a "tetranucleosomes-on-a-string" extended structure; then the tetranucleosomal unit unfolds to a complete open nucleosomal array in one or two steps. (Adapted, with permission, from Li et al. 2016.) 
have been resolved recently (Song et al. 2014; Li et al. 2016). We showed that mechanical unfolding of a $30-\mathrm{nm}$ fiber is a multistep process (Fig. 2B). Under increasing tensile force applied, the compacted chromatin fiber first unfolds to a "tetranucleosomes-on-a-string" extended structure by disrupting the internucleosome interactions between tetranucleosomal units. The tetranucleosomal unit exists as a stable structural intermediate of the 30$\mathrm{nm}$ chromatin fiber and further unfolds to a more extended "beads-on-a-string" conformation by disrupting the nucleosome-nucleosome interactions within the tetranucleosomal unit in a two-step, three-transition-state process at force of $\sim 3.5 \mathrm{pN}$, which is characteristic of three transitions with the step sizes of $1 L, 2 L$, and $3 L$ (in which $L$ is the linker length between the adjacent nucleosomes), respectively (Fig. 2C,D). Further increasing force causes nucleosome unwrapping in two major stages, including the one-step disruption of the outer DNA turn and the one-step unwrapping of the inner turn, consistent with previous investigations (Brower-Toland et al. 2002; Hall et al. 2009; Bintu et al. 2012; Li et al. 2016). Importantly, similar dynamic processes can be observed in the chromatin fibers assembled not only on regular tandem repeat of the 601 DNA sequence but also on the scrambled (nonrepetitive) DNA sequence, suggesting that the existence of tetranucleosomal units is not dependent on DNA sequence. Moreover, the low energy required to disrupt the interaction between two tetranucleosomal units $\left(\sim 1.8 k_{\mathrm{B}} T\right)$, which is comparable to the thermal fluctuations, suggests that chromatin fibers may undergo spontaneously rapid folding/unfolding dynamics between a compact regular 30-nm chromatin fiber and an extended "tetranucleosomes-on-a-string" at physiological conditions in vivo. Thus, it is likely that the chromatin fibers in vivo probably adopt an irregular "tetranucleosomes-on-a-string" structure, which combines fully folded regular zigzag tetranucleosomal clusters/ stacks with partially unfolded "nucleosomal array" or "nucleosomal clutches" regions (Collepardo-Guevara and Schlick 2011).

\section{EPIGENETIC REGULATION OF CHROMATIN FIBERS BY CHROMATIN FACTORS}

In the past 20 years, many chromatin factors, including chromatin modifications, histone chaperones, histone variants, chromatin remodelers, and chromatin architectural proteins, have been shown to be involved in regulating chromatin dynamics (Luger and Hansen 2005; Hake and Allis 2006; Greaves et al. 2007). Deciphering the structure and dynamics of the $30-\mathrm{nm}$ chromatin fibers in molecular details is essential for understanding such regulations. Our cryo-EM structures clearly imply the presence of three important interaction interfaces in the 30-nm fiber-namely, DNA-histone interaction interfaces within the nucleosome, internucleosome interaction interfaces within the tetranucleosomal unit, and interaction interfaces between tetranucleosomal units, which can be potentially regulated by different chromatin factors (Fig. 3). The newly developed in vitro single-molecule techniques and in vivo genomic approaches enable us to investigate the epigenetic regulations of 30-nm fibers by different chromatin factors (Hsieh et al. 2015; Li et al. 2016; Risca et al. 2017).

Previously, FACT, a conserved histone chaperone for H2A-H2B dimers, has been shown to destabilize nucleosomes for RNA polymerase progression on chromatin templates (Orphanides et al. 1998, 1999; Belotserkovskaya et al. 2003) and maintain chromatin structure in vivo during DNA transcription, replication, and repair (Saunders et al. 2003; Fujimoto et al. 2012; Formosa 2013; McCullough et al. 2015). However, the molecular mechanisms of how FACT remodels chromatin remain largely unclear. As discussed above, the nucleosomal stacks within tetranucleosomal unit are mainly stabilized by the interactions between the $\mathrm{H} 2 \mathrm{~B}-$ helix $\alpha 1 / \alpha \mathrm{C}$ and the adjacent $\mathrm{H} 2 \mathrm{~A}-$ helix $\alpha 2$ (Song et al. 2014). A recent structural study revealed that the recognition of $\mathrm{H} 2 \mathrm{~A}-\mathrm{H} 2 \mathrm{~B}$ heterodimer by FACT is mainly mediated by the interactions between the U-turn motif of Spt16M and the amino-terminal $\alpha 1$ helix of H2B (Hondele et al. 2013), suggesting that
A

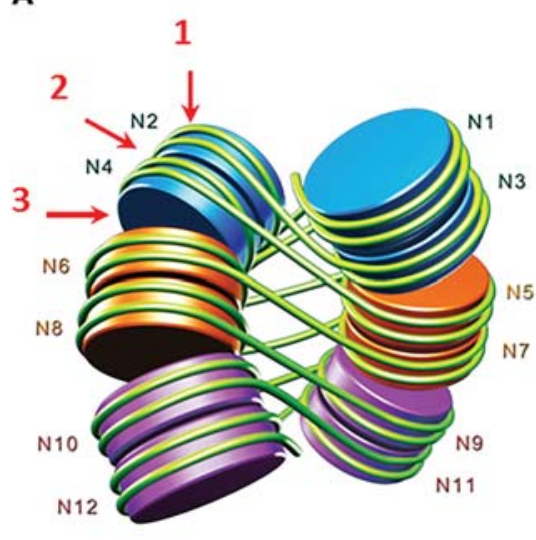

B

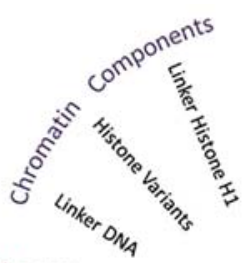

Nucleosomal array

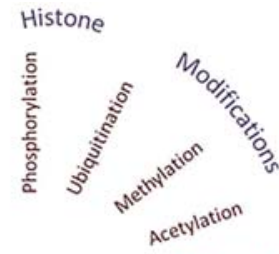

30-nm chromatin fiber
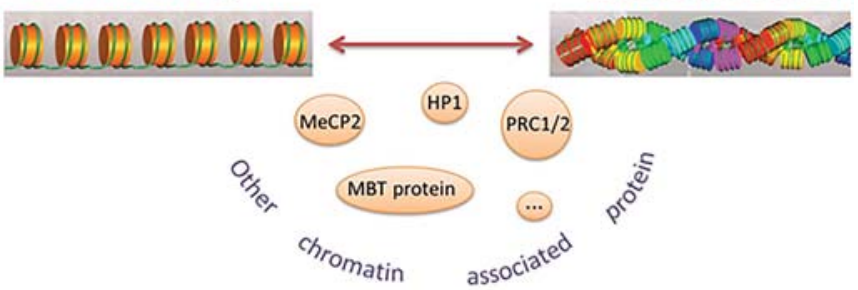

Figure 3. Epigenetic regulation of chromatin fibers by chromatin factors. (A) Schematic model of 30-nm chromatin fibers with the three important interaction interfaces as indicated by arrows, including the DNA-histone interaction interfaces within nucleosome (1), internucleosome interaction interfaces within tetranucleosomal unit (2), and interaction interfaces between tetranucleosomal units (3). (B) The different chromatin factors potentially regulate the interaction interfaces of 30 -nm chromatin fibers. 
FACT may remodel chromatin fibers via interfering these interfacial interactions. Indeed, our single-molecule measurements showed that FACT can remodel higher-order chromatin structure by destabilizing the tetranucleosomal unit (Li et al. 2016). More importantly, genomic analyses showed that FACT destabilizes the N/N+2 Micro-C interactions (tetranucleosomal motifs) to facilitate gene transcription in yeast. Distinct domains of FACT have been shown to interact with $\mathrm{H} 2 \mathrm{~A} / \mathrm{H} 2 \mathrm{~B}$ or $\mathrm{H} 3 / \mathrm{H} 4$ separately (Stuwe et al. 2008; Zhang et al. 2015; Tsunaka et al. 2016), suggesting that these distinct domains of FACT might be responsible for their different activities in remodeling the nucleosome and/or the tetranucleosomal unit. It will be of great interest to generate a series of FACT mutants to resolve the distinctive activity in remodeling the nucleosome and/or the tetranucleosome using singlemolecule measurements. Beside FACT, several other chromatin remodelers (such as INO80, which recognizes the H2B $\alpha \mathrm{C}$ helix) (Tosi et al. 2013), or certain repressive chromatin factors (such as PRC1, which has been shown to compact three to four nucleosomes) (Francis et al. 2004) may also fulfill their biological functions via modulating the dynamics or stability of tetranucleosomal units. The tetranucleosomal unit may provide an additional level of gene regulation beyond the nucleosome. It will be of great interest to identify these factors and decipher their regulatory interactions with the tetranucleosomal units.

The incorporation of histone variants has been shown to regulate the structure and dynamics of chromatin fibers and create architecturally distinct chromatin states that play diverse functions in genome-associated biological processes. Among them, histone variant H3.3 has been largely considered as a mark of transcriptionally activated genes and has been deposited into transcribed genes, promoters, and gene-regulatory elements (Schwartz and Ahmad 2005; Jin et al. 2009; Goldberg et al. 2010). However, a few recent studies showed that H3.3 was also incorporated at regions of the genome that are typically thought to be relatively transcriptionally inactive, such as the telomere and pericentric heterochromatin in mouse embryonic stem (mES) cells (van der Heijden et al. 2007; Goldberg et al. 2010; Wong et al. 2010). H3.3 differs from canonical $\mathrm{H} 3$ at only four amino acid residues, three are hidden inside the NCP in region 87-90 and one residue, Ser31, is exposed outside of the NCP. We and others have showed that Ala87 and Gly90 are the principal determinants of human H3.3 specificity in DAXX recognition (Elsässer et al. 2012; Liu et al. 2012). We also investigated how H3.3 regulates the nucleosome/chromatin dynamics and gene transcription (Fig. 4; Chen et al. 2013). Our FRET and magnetic tweezers experiments showed that H3.3 has little effect on the stability of nucleosomes, which was consistent with previous findings (Flaus et al. 2004; Thakar et al. 2009), but it greatly impairs the compaction of chromatin by analytical ultracentrifugation (AUC) and EM analyses (Chen et al. 2013). Interestingly, we also showed that the histone variant H2A.Z not only stabilizes the nucleosome but also facilitates compaction of nucleosomal arrays into a featured "ladder-liker" chromatin fiber. Although the incorporation of H3.3 does not affect the stabilization effect of H2A.Z on nucleosomes, it counteracts H2A.Z-mediated chromatin compaction (Fig. 4A,B). Residues 89 and 90 of H3.3 were mainly responsible for the counteractivity of H2A.Z-mediated chromatin compaction. Moreover, we found that H3.3 could antagonize the inhibitory effects of H2A.Z on chromatin transcription in vitro by RNA Pol II, which partially resulted from the counteractivity of compaction by H2A.Z. Our results suggest that H3.3 may play a dominant role in regulation of the dynamics of higher-ordered chromatin and transcriptional activity in the chromatin context. To this end, we further analyzed the dynamic depositions and/ or replacement of $\mathrm{H} 2 \mathrm{~A} . \mathrm{Z}$ and $\mathrm{H} 3.3$ and the corresponding structural changes of chromatin at the enhancer and promoter regions of RAR/RXR targeted genes during gene activation by all-trans-retinoid acid (tRA) induction. Our results (Fig. 4C) revealed that deposition of $\mathrm{H} 2 \mathrm{~A} . \mathrm{Z}$ results in compaction of chromatin at promoter regions, which inhibits the transcription of the Cyp26al and Hoxal genes before tRA induction, whereas the incorporation of $\mathrm{H} 3.3$ at enhancer regions decorates the chromatin architecture to a relatively open conformation, allowing the recognition and binding of transcriptional activators, which subsequently recruits the ATP-dependent chromatin remodeling complexes and/or histone-modifying enzymes to remodel the nucleosome architecture at promoter regions upon gene induction, as previously reported (Li et al. 2010). Our results provide new insights into the molecular mechanism of how histone variants function cooperatively to establish featured chromatin structures at enhancer and promoter regions to prime inducible genes for rapidly activation in response to environmental stimulation.

The centromere-specific histone $\mathrm{H} 3$ variant $(\mathrm{CenH} 3$ [CENP-A in humans]), is an epigenetic factor essential for the centromere identity and function (Allshire and Karpen 2008). In human cells, CENP-A is specifically recognized and deposited into centromeres by its chaperone and assembly factor HJURP (Dunleavy et al. 2009; Foltz et al. 2009). Our structural and biochemical analysis showed that other than the CATD of CENP-A, previously identified as the exclusive region responsible for HJURP binding (Black et al. 2004; Bassett et al. 2012), the residue Ser68 also plays essential roles for HJURP binding ( $\mathrm{Hu}$ et al. 2011). We further showed that the dynamic phosphorylation/dephosphorylation of Ser68 in CENP-A, which is mediated by the $\mathrm{Cdk} 1 / \mathrm{Cyclin} \mathrm{B}$ and PP $1 \alpha$ complex, temporally controls the HJURP-mediated assembly of CENPA into centromeric regions (Yu et al. 2015). CENP-N, an indispensable member of CCAN (the constitutive centromere-associated network), was identified as the first "reader" of epigenetic marks present in the CENP-A-containing chromatin (Carroll et al. 2009). CENP-N is dynamically recruited to centromeres/kinetochores during the cell cycle (Hellwig et al. 2011) and plays an essential role in the assembly of kinetochore complex at centromeres and faithful segregation of sister chromatids during cell division (Foltz et al. 2006; Carroll et al. 2009). By using biochemical, biophysical, and cell-based assays, we showed that the RG loop, the two CENP-A-specific residues Arg80 and Gly81 in loop1 located at the lateral surface of CENP-A 
A
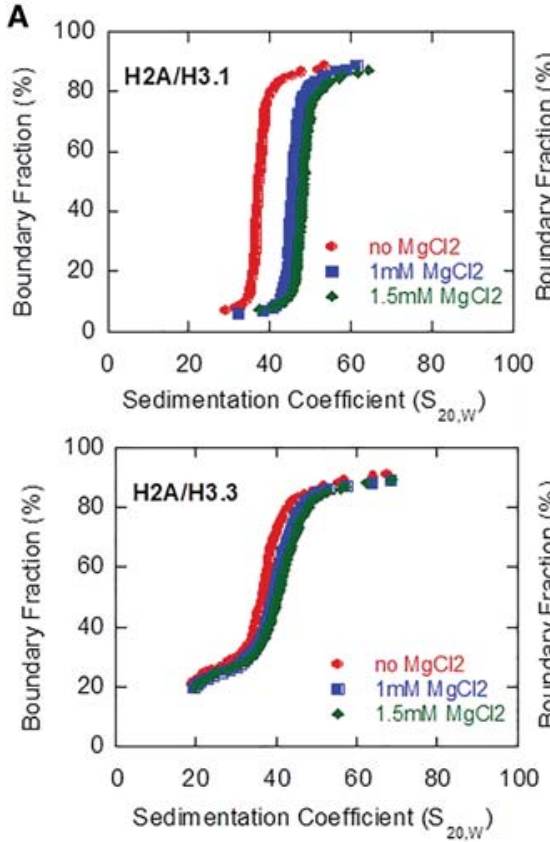

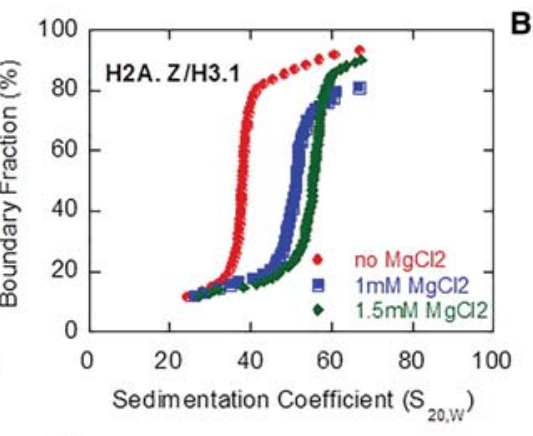

B $\mathrm{H} 2 \mathrm{~A} / \mathrm{H} 3.1$

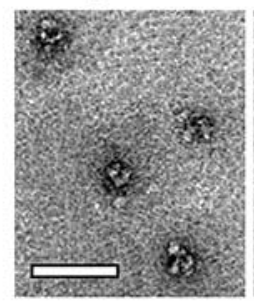

H2A.Z/H3.1

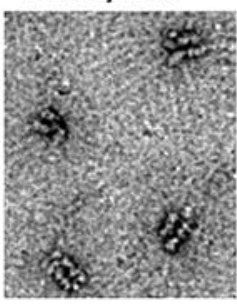

H2A/H3.3

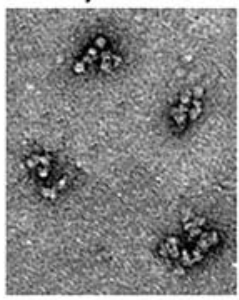

H2A.Z/H3.3

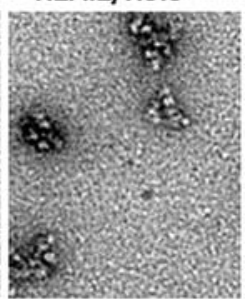

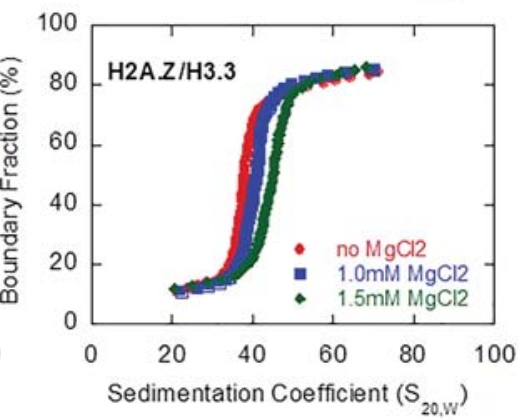

C
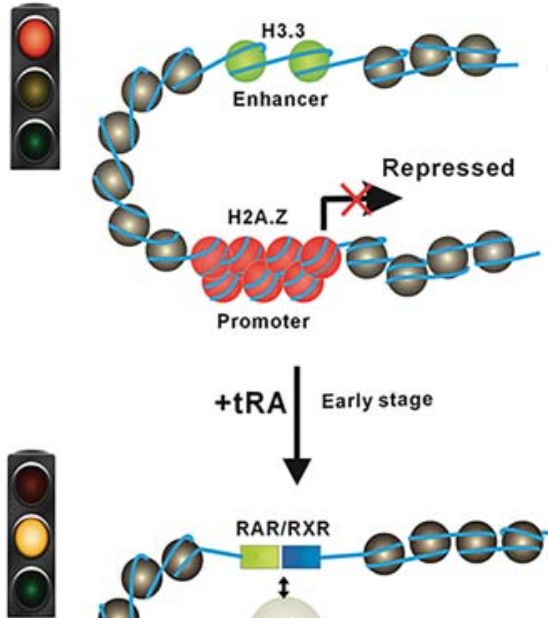

H3.1/H2A nucleosome

H3.1/H2A.Z nucleosome

H3.3/H2A.Z nucleosome

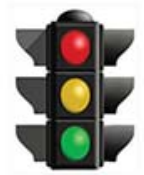

H3.3/H2A nucleosome

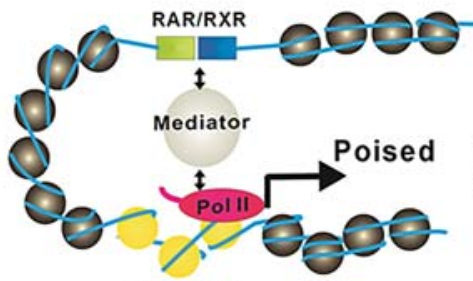

H2A.Z/H3.3
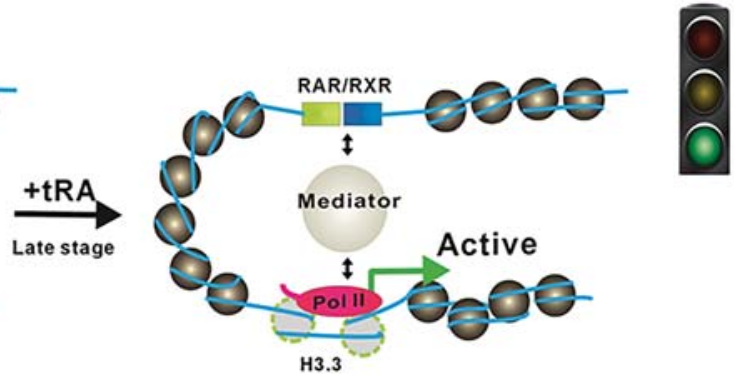

Figure 4. Histone variants H3.3 and H2A.Z regulate chromatin structures cooperatively for gene activation. $(A)$ Sedimentation coefficient distribution plots for the canonical, H2A.Z-, H3.3-, and double-variant H2A.Z/H3.3-containing nucleosomal arrays at 0, 1.0, and $1.5 \mathrm{mM} \mathrm{MgCl}_{2}$. (B) Negatively stained electron microscopic (EM) images of the canonical, H2A.Z-, H3.3-, and double-variant H2A.Z/ H3.3-containing chromatin fibers in $1.0 \mathrm{mM} \mathrm{MgCl}_{2}$. Scale bar, $100 \mathrm{~nm}$. (C) The model for the dynamic regulation of H2A.Z and H3.3 on chromatin structures at the enhancer and promoter regions during the gene activation process by the addition of trans-retinoic acid (tRA). (Adapted, with permission, from Chen et al. 2013.)

nucleosome (Tachiwana et al. 2011), not only provides the recognition site for the binding of CENP-N to the CENP-A nucleosome, but also facilitates the folding of CENP-A arrays into a compact "ladder-like" chromatin structure in the presence of $\mathrm{MgCl}_{2}$, as revealed by AUC and EM analyses (Fig. 5A-C; Fang et al. 2015). Interestingly, we found that the formation of a compact "ladder-like" structure of CENP-A chromatin inhibits the binding and recruitment of
CENP-N through concealing/hiding the RG loop within chromatin fiber. More importantly, our fluorescence resonance energy transfer (FRET) analysis and SNAP imaging showed that upon $\mathrm{G}_{1} / \mathrm{S}$ phase transition, centromeric chromatin switches from the compact to an open state because of the dilution of CENP-A nucleosome during DNA replication, which enables the now-exposed RG loop to recruit CENP-N at the $\mathrm{S}$ phase (Fig. 5D). 
A

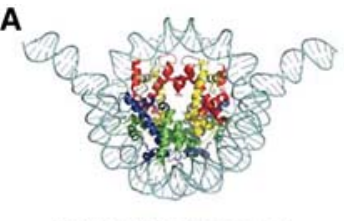

CENP-A Nucleosome

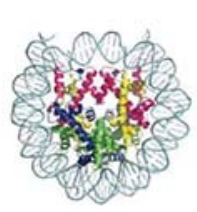

H3 Nucleosome

C
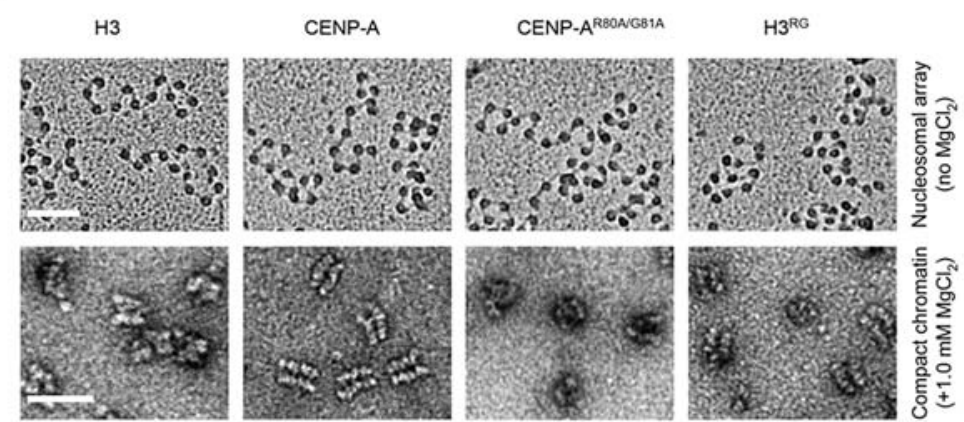

D

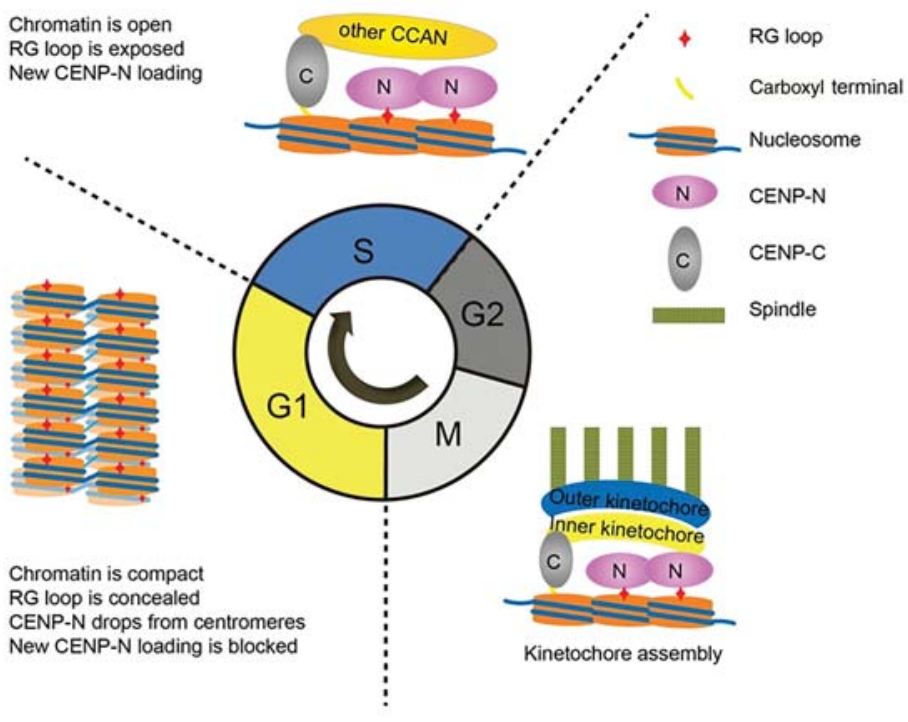

Figure 5. Histone variant CENP-A regulates centromeric chromatin structures for cell-dependent recruitment of CENP-N. $(A)$ The comparison between the X-ray structures of nucleosomes containing either CENP-A or canonical H3 (top) and the side view of CENP-A nucleosome (bottom) with the CENP-A molecule shown in magenta and superimposition of H3 loop1 regions (orange). Arrows indicate the tip of the CENP-A loop1 containing Arg80 and Gly81 residues (green). ClustalX2 alignment of the CATD domain is shown with residues R80G81 highlighted (red) in CENP-A orthologs from Homo sapiens (Hs), Pongo abelii (Pa), Bos taurus (Bt), and Mus musculus $(\mathrm{Mm})$, and the corresponding region in human $\mathrm{H} 3$. (B) Distribution plots of $S_{\text {ave }}$ values of chromatins containing canonical H3, CENP-A, CENP-A $\mathrm{R}^{\mathrm{R} 0 \mathrm{~A} / \mathrm{G} 81 \mathrm{~A}}$, and $\mathrm{H}^{\mathrm{RG}}$ at different concentration of $\mathrm{MgCl}_{2} .(C)$ EM images of the canonical H3, CENP-A, CENP$\mathrm{A}^{\mathrm{R} 80 \mathrm{~A} / \mathrm{G} 81 \mathrm{~A}}$, and $\mathrm{H}^{\mathrm{RG}}$ containing nucleosomal arrays (by the metal-shadowing method; scale bar, $50 \mathrm{~nm}$ ) and their corresponding compact states in $1.0 \mathrm{mM} \mathrm{MgCl}_{2}$ (by the negatively stained method; scale bar, $100 \mathrm{~nm}$ ). $(D)$ The model of structural transitions of centromeric chromatin regulate the cell cycle-dependent recruitment of CENP-N via modulating the accessibility of the RG loop of CENP-A. (Adapted, with permission, from Fang et al. 2015.)

\section{ORGANIZATION OF CHROMATIN FIBERS IN NUCLEUS}

The 30-nm chromatin fiber has long been thought to be the first level of the hierarchical chromatin compaction, but the existence of the 30-nm fiber in vivo still remains controversial. Studies of purified or reconstituted chromatin have provided evidence supporting the existence of longitudinally compacted 30-nm chromatin fibers; however, until now high-resolution imaging of chromatin in living cells had not been possible (Eltsov et al. 2008; Fussner et al. 2011a). Early cryo-EM studies have shown that a $30-\mathrm{nm}$ fiber is indeed the most predominant form of starfish sperm and nucleated chicken erythrocyte chromatin (Horowitz et al. 1994; Woodcock 1994; Scheffer et al.
2011). Using cryo-EM tomography of vitreous sections, Frangakis and colleagues showed that the most predominant form of chromatin in chicken erythrocyte nuclei is indeed a 30 -nm fiber arranged in a two-start helix formation with $\sim 6.7$ nucleosomes per turn, in which the nucleosomes are juxtaposed face to face (Scheffer et al. 2011). In addition, the stacked nucleosomes were shown to be shifted off their superhelical axes, with an axial translation of $\sim 3.4 \mathrm{~nm}$ and an azimuthal rotation of $\sim 54^{\circ}$, very consistent with the high-resolution structure of in vitro reconstituted chromatin fibers (Schalch et al. 2005; Song et al. 2014). In addition, Rando and colleagues recently developed a novel Hi-C-based method named "Micro-C" to probe nucleosome organization at nucleosome resolution in yeast (Hsieh et al. 2015). Despite the lack of periodicity 
in their data set, they showed that $\mathrm{N} / \mathrm{N}+2$ and $\mathrm{N} / \mathrm{N}+1$ nucleosome pairs are similarly abundant in the whole genome, which suggests the wide existence of tetranucleosomal folding motifs in yeast genome. Similarly, using the EM-assisted nucleosome interaction capture (EMANIC) cross-linking experiments in combination with mesoscale modeling of chromatin fibers, Schlick and colleagues showed a dominant relaxed two-start zigzag organization rather than longitudinal compaction associated with the 30-nm fiber (Grigoryev et al. 2009). Most recently, combining the ionizing radiation-induced spatially correlated cleavage of DNA with deep-sequencing technique (RICC-seq), Greenleaf and colleagues provided the first genome-wide map of the chromatin secondary structure in living human cells at the one- to three-nucleosome (50- to 500-bp) scale (Risca et al. 2017). Unbiased analysis of RICC-seq signals in intact interphase nuclei reveals that RICC-seq fragmentation patterns in $\mathrm{H} 3 \mathrm{~K} 9 \mathrm{me} 3-$ and $\mathrm{H} 3 \mathrm{~K} 27$ me3-marked heterochromatin regions are consistent with variable longitudinal chromatin compaction of two-start helical fibers with face-to-face stacked alternating nucleosomes in tri- or tetranucleosome units, as seen in the structure of reconstituted chromatin fiber (Song et al. 2014).

This overwhelming body of experimental evidences from EM and genomic studies strongly support that twostart helical fibers with stacked alternating nucleosomes are an important mechanism for generating chromatin compaction both in vitro and in vivo. However, cryoEM (Gan et al. 2013), X-ray scattering (Nishino et al. 2012; Maeshima et al. 2014), and electron spectroscopy imaging (ESI) studies (Bazett-Jones et al. 2008; Fussner et al. 2011b, 2012) of the nucleus do not support the existence of the regular $30-\mathrm{nm}$ fiber within intact cells (Eltsov et al. 2008; Fussner et al. 2011a,b; Nishino et al. 2012). Because of the poor contrast of DNA in vitreous ice and the limited 3D sampling volume of ESI, it is technically difficult to identify chromatin unambiguously and to reconstruct $3 \mathrm{D}$ organization of chromatin through large nuclear volume in cryo-EM experiments. Most recently, O'Shea and colleagues developed a novel ChromEMT technique by combining electron microscopy tomography (EMT) with a labeling method, which explores a fluorescent dye (DRAQ5) that binds to DNA and enhances the contrast of DNA, enabling chromatin to be visualized with $\mathrm{OsO}_{4}$ in $\mathrm{EM}$ (Ou et al. 2017). Using this technique, they only observed a flexible and disordered granular chromatin chain with diameters between 5 and $24 \mathrm{~nm}$, but no regular higher-order chromatin fibers in human interphase and mitotic cells in situ. However, a few important issues still need to be clarified in the technique. The first is whether DRAQ5 also binds to other type nucleic acids in situ, such as RNA (particularly double-stranded RNA [dsRNA]). It is unclear how to distinguish chromatin fibers from other nucleic acid-protein particles, which are abundant and distributed widely in nuclei. Second, no evidence in vitro or in vivo showed that DRAQ5 can bind equally to free DNA and chromatin DNA. It is likely that the DNA in compacted chromatin fragments may not be accessible for staining by the dye. In addition, the path of DNA in the chromatin fiber cannot be directly observed in vivo by the ChromEMT technique under such low resolution. As discussed above, we and others showed that chromatin fibers mainly adopt a two-started helical conformation with alternating nucleosomes stacked face to face in vitro and in vivo, in which the two nucleosomal stacks are connected by linker DNA and distanced from each by $\sim 7.5-10 \mathrm{~nm}$ (Bednar et al. 1998; Song et al. 2014; Li et al. 2016). In this context, when the linker DNA cannot be discriminated, only two separated nucleosomal stacks with a diameter of $\sim 10 \mathrm{~nm}$ can be actually observed in ChromEMT. Moreover, extensive fixation by chemical cross-linking and dehydration by ethanol during sample preparation in ChromEMT may damage the ultrastructure and $3 \mathrm{D}$ organization of chromatin fiber. Therefore, it is still a big challenge to preserve the natural $3 \mathrm{D}$ conformation of chromatin fiber in ChromEMT or cryo-EM tomography.

\section{PERSPECTIVES AND CONCLUSION}

Our $3 \mathrm{D}$ cryo-EM structures at $11 \AA$ resolution have provided the fundamental structural features of the elusive 30-nm chromatin fiber and a solid foundation for understanding the basic principle of chromatin compaction, whereas higher-resolution structures of chromatin fiber are needed to uncover much more structural details for the nucleosome-nucleosome, nucleosome-H1, and H1H1 interactions in chromatin fibers. In addition, our cryoEM structures clearly imply the presence of three important interaction interfaces in the $30-\mathrm{nm}$ fiber, whereas it still remains unclear how these interfaces are regulated by different chromatin factors. Regarding the variation of NRLs in vivo, reconstituted chromatin fibers with a combination of different NRLs will also be good candidates for single-molecule and cryo-EM studies in the future. These further studies will not only enhance our understanding of the diversity of chromatin structures in vivo but also provide structural basis for how different combinations of DNA sequences, NRLs, histone variants, chromatin modifications, and chromatin architectural proteins can be coordinated to precisely regulate the biological function of genomic DNA in the nucleus.

It is still a puzzle as to whether the structural results from the in vitro studies can represent the actual structure of chromatin fibers in situ. Therefore, the 3D organization of chromatin fibers in the intact nuclei needs to be further studied by using newly developed techniques. Well-characterized chromatin fibers reconstituted in vitro, including compacted 30-nm fibers and open nucleosomal arrays, would be perfect structural references for analyzing the 3D organization of chromatin fibers in situ in these studies. The combination of cryo-EM with super-resolution fluorescence imaging techniques has been recently developed to visualize and quantify the ultrastructure of cryopreserved cells (Chang et al. 2014; Liu et al. 2015). The combination of genomic approaches (such as micro-C and RICC-seq) and CRISPR (clustered regularly interspaced short palindromic repeat)-based imaging techniques may 
enable us to probe the ultrastructure and 3D organization of chromatin fiber at defined genomic regions in the intact nuclei. Undoubtedly, more structural detail for the 3D organization of chromatin fibers in situ can be obtained by the application of these advanced imaging techniques in the future.

\section{ACKNOWLEDGMENTS}

This work was supported by grants to G.L. from the National Natural Science Foundation of China (31630041, 31525013, and 31521002), the Ministry of Science and Technology of China (2017YFA0504200, 2015CB856200), and the Chinese Academy of Sciences (CAS) Strategic Priority Research Program (XDB1904 0202); and to P.C. from the National Natural Science Foundation of China (31471218) and the Youth Innovation Promotion Association CAS (2015071). The work was also supported by the CAS Key Research Program on Frontier Science (QYZDY-SSW-SMC020) and HHMI international research scholar grant (55008737) for G.L. The authors declare that there are no conflicts of interest.

\section{REFERENCES}

Allan J, Hartman PG, Crane-Robinson C, Aviles FX. 1980. The structure of histone $\mathrm{H} 1$ and its location in chromatin. Nature 288: 675-679.

Allshire RC, Karpen GH. 2008. Epigenetic regulation of centromeric chromatin: Old dogs, new tricks? Nat Rev Genet 9: 923 937.

Bassett EA, DeNizio J, Barnhart-Dailey MC, Panchenko T, Sekulic N, Rogers DJ, Foltz DR, Black BE. 2012. HJURP uses distinct CENP-A surfaces to recognize and to stabilize CENP-A/histone H4 for centromere assembly. Dev Cell 22: 749-762.

Bates DL, Thomas JO. 1981. Histones H1 and H5: One or two molecules per nucleosome? Nucleic Acids Res 9: 5883-5894.

Bazett-Jones DP, Li R, Fussner E, Nisman R, Dehghani H. 2008. Elucidating chromatin and nuclear domain architecture with electron spectroscopic imaging. Chromosome Res 16: 397412

Bednar J, Horowitz RA, Grigoryev SA, Carruthers LM, Hansen JC, Koster AJ, Woodcock CL. 1998. Nucleosomes, linker DNA, and linker histone form a unique structural motif that directs the higher-order folding and compaction of chromatin. Proc Natl Acad Sci 95: 14173-14178.

Belotserkovskaya R, Oh S, Bondarenko VA, Orphanides G, Studitsky VM, Reinberg D. 2003. FACT facilitates transcriptiondependent nucleosome alteration. Science 301: 1090-1093.

Bintu L, Ishibashi T, Dangkulwanich M, Wu YY, Lubkowska L, Kashlev M, Bustamante C. 2012. Nucleosomal elements that control the topography of the barrier to transcription. Cell 151: 738-749.

Black BE, Foltz DR, Chakravarthy S, Luger K, Woods VL Jr, Cleveland DW. 2004. Structural determinants for generating centromeric chromatin. Nature 430: 578-582.

Brower-Toland BD, Smith CL, Yeh RC, Lis JT, Peterson CL, Wang MD. 2002. Mechanical disruption of individual nucleosomes reveals a reversible multistage release of DNA. Proc Natl Acad Sci 99: 1960-1965.

Carroll CW, Silva MC, Godek KM, Jansen LE, Straight AF. 2009. Centromere assembly requires the direct recognition of CENP-A nucleosomes by CENP-N. Nat Cell Biol 11: 896902.

Chang YW, Chen S, Tocheva EI, Treuner-Lange A, Löbach S, Sogaard-Andersen L, Jensen GJ. 2014. Correlated cryogenic photoactivated localization microscopy and cryo-electron tomography. Nat Methods 11: 737-739.

Chen P, Zhao J, Wang Y, Wang M, Long H, Liang D, Huang L, Wen Z, Li W, Li X, et al. 2013. H3.3 actively marks enhancers and primes gene transcription via opening higher-ordered chromatin. Genes Dev 27: 2109-2124.

Collepardo-Guevara R, Schlick T. 2011. The effect of linker histone's nucleosome binding affinity on chromatin unfolding mechanisms. Biophys J 101: 1670-1680.

Cui Y, Bustamante C. 2000. Pulling a single chromatin fiber reveals the forces that maintain its higher-order structure. Proc Natl Acad Sci 97: 127-132.

Cutter AR, Hayes JJ. 2015. A brief review of nucleosome structure. FEBS Lett 589: 2914-2922.

Dorigo B, Schalch T, Kulangara A, Duda S, Schroeder RR, Richmond TJ. 2004. Nucleosome arrays reveal the two-start organization of the chromatin fiber. Science 306: 1571-1573.

Dunleavy EM, Roche D, Tagami H, Lacoste N, Ray-Gallet D, Nakamura Y, Daigo Y, Nakatani Y, Almouzni-Pettinotti G. 2009. HJURP is a cell-cycle-dependent maintenance and deposition factor of CENP-A at centromeres. Cell 137: 485-497.

Elsässer SJ, Huang H, Lewis PW, Chin JW, Allis CD, Patel DJ. 2012. DAXX envelops a histone H3.3-H4 dimer for H3.3specific recognition. Nature 491: 560-565.

Eltsov M, MacLellan KM, Maeshima K, Frangakis AS, Dubochet J. 2008. Analysis of cryo-electron microscopy images does not support the existence of 30-nm chromatin fibers in mitotic chromosomes in situ. Proc Natl Acad Sci 105: 19732 19737.

Fang J, Liu Y, Wei Y, Deng W, Yu Z, Huang L, Teng Y, Yao T, You Q, Ruan H, et al. 2015. Structural transitions of centromeric chromatin regulate the cell cycle-dependent recruitment of CENP-N. Genes Dev 29: 1058-1073.

Finch JT, Klug A. 1976. Solenoidal model for superstructure in chromatin. Proc Natl Acad Sci 73: 1897-1901.

Flaus A, Rencurel C, Ferreira H, Wiechens N, Owen-Hughes T. 2004. Sin mutations alter inherent nucleosome mobility. EMBO J 23: 343-353.

Foltz DR, Jansen LE, Black BE, Bailey AO, Yates JR III, Cleveland DW. 2006. The human CENP-A centromeric nucleosome-associated complex. Nat Cell Biol 8: 458-469.

Foltz DR, Jansen LE, Bailey AO, Yates JR III, Bassett EA, Wood S, Black BE, Cleveland DW. 2009. Centromere-specific assembly of CENP-a nucleosomes is mediated by HJURP. Cell 137: 472-484.

Formosa T. 2013. The role of FACT in making and breaking nucleosomes. Biochim Biophys Acta 1819: 247-255.

Francis NJ, Kingston RE, Woodcock CL. 2004. Chromatin compaction by a polycomb group protein complex. Science 306: $1574-1577$

Fujimoto M, Takaki E, Takii R, Tan K, Prakasam R, Hayashida N, Iemura S, Natsume T, Nakai A. 2012. RPA assists HSF1 access to nucleosomal DNA by recruiting histone chaperone FACT. Mol Cell 48: 182-194.

Fussner E, Ching RW, Bazett-Jones DP. 2011a. Living without 30 nm chromatin fibers. Trends Biochem Sci 36: 1-6.

Fussner E, Djuric U, Strauss M, Hotta A, Perez-Iratxeta C, Lanner F, Dilworth FJ, Ellis J, Bazett-Jones DP. 2011b. Constitutive heterochromatin reorganization during somatic cell reprogramming. EMBO J 30: 1778-1789.

Fussner E, Strauss M, Djuric U, Li R, Ahmed K, Hart M, Ellis J, Bazett-Jones DP. 2012. Open and closed domains in the mouse genome are configured as 10-nm chromatin fibres. EMBO Rep 13: 992-996.

Gan L, Ladinsky MS, Jensen GJ. 2013. Chromatin in a marine picoeukaryote is a disordered assemblage of nucleosomes. Chromosoma 122: 377-386.

Gerchman SE, Ramakrishnan V. 1987. Chromatin higher-order structure studied by neutron scattering and scanning transmission electron microscopy. Proc Natl Acad Sci 84: 7802-7806.

Ghirlando R, Felsenfeld G. 2008. Hydrodynamic studies on defined heterochromatin fragments support a 30-nm fiber having six nucleosomes per turn. J Mol Biol 376: 1417-1425. 
Goldberg AD, Banaszynski LA, Noh KM, Lewis PW, Elsaesser SJ, Stadler S, Dewell S, Law M, Guo X, Li X, et al. 2010. Distinct factors control histone variant H3.3 localization at specific genomic regions. Cell 140: 678-691.

Greaves IK, Rangasamy D, Ridgway P, Tremethick DJ. 2007. H2A.Z contributes to the unique 3D structure of the centromere. Proc Natl Acad Sci 104: 525-530.

Grigoryev SA, Arya G, Correll S, Woodcock CL, Schlick T. 2009. Evidence for heteromorphic chromatin fibers from analysis of nucleosome interactions. Proc Natl Acad Sci 106: 13317-13322.

Hake SB, Allis CD. 2006. Histone H3 variants and their potential role in indexing mammalian genomes: The "H3 barcode hypothesis". Proc Natl Acad Sci 103: 6428-6435.

Hall MA, Shundrovsky A, Bai L, Fulbright RM, Lis JT, Wang MD. 2009. High-resolution dynamic mapping of histoneDNA interactions in a nucleosome. Nat Struct Mol Biol 16: 124-129.

Hellwig D, Emmerth S, Ulbricht T, Döring V, Hoischen C, Martin R, Samora CP, McAinsh AD, Carroll CW, Straight AF, et al. 2011. Dynamics of CENP-N kinetochore binding during the cell cycle. J Cell Sci 124: 3871-3883.

Hondele M, Stuwe T, Hassler M, Halbach F, Bowman A, Zhang ET, Nijmeijer B, Kotthoff C, Rybin V, Amlacher S, et al. 2013. Structural basis of histone H2A-H2B recognition by the essential chaperone FACT. Nature 499: 111-114.

Horowitz RA, Agard DA, Sedat JW, Woodcock CL. 1994. The three-dimensional architecture of chromatin in situ: Electron tomography reveals fibers composed of a continuously variable zig-zag nucleosomal ribbon. J Cell Biol 125: 1-10.

Hsieh TH, Weiner A, Lajoie B, Dekker J, Friedman N, Rando OJ. 2015. Mapping nucleosome resolution chromosome folding in yeast by Micro-C. Cell 162: 108-119.

Hu H, Liu Y, Wang M, Fang J, Huang H, Yang N, Li Y, Wang J, Yao X, Shi Y, et al. 2011. Structure of a CENP-A-histone H4 heterodimer in complex with chaperone HJURP. Genes Dev 25: 901-906.

Jin C, Zang C, Wei G, Cui K, Peng W, Zhao K, Felsenfeld G. 2009. H3.3/H2A.Z double variant-containing nucleosomes mark "nucleosome-free regions" of active promoters and other regulatory regions. Nat Genet 41: 941-945.

Kruithof M, Chien FT, Routh A, Logie C, Rhodes D, van Noort J. 2009. Single-molecule force spectroscopy reveals a highly compliant helical folding for the 30-nm chromatin fiber. Nat Struct Mol Biol 16: 534-540.

Langmore JP, Paulson JR. 1983. Low angle x-ray diffraction studies of chromatin structure in vivo and in isolated nuclei and metaphase chromosomes. J Cell Biol 96: 1120-1131.

Li G, Reinberg D. 2011. Chromatin higher-order structures and gene regulation. Curr Opin Genet Dev 21: 175-186.

Li G, Margueron R, Hu G, Stokes D, Wang YH, Reinberg D. 2010. Highly compacted chromatin formed in vitro reflects the dynamics of transcription activation in vivo. Mol Cell 38: 41-53.

Li W, Chen P, Yu J, Dong L, Liang D, Feng J, Yan J, Wang PY, Li Q, Zhang Z, et al. 2016. FACT remodels the tetranucleosomal unit of chromatin fibers for gene transcription. Mol Cell 64: $120-133$.

Liu CP, Xiong C, Wang M, Yu Z, Yang N, Chen P, Zhang Z, Li G, $\mathrm{Xu}$ RM. 2012. Structure of the variant histone H3.3-H4 heterodimer in complex with its chaperone DAXX. Nat Struct Mol Biol 19: 1287-1292.

Liu B, Xue Y, Zhao W, Chen Y, Fan C, Gu L, Zhang Y, Zhang X, Sun L, Huang X, et al. 2015. Three-dimensional super-resolution protein localization correlated with vitrified cellular context. Sci Rep 5: 13017.

Luger K, Hansen JC. 2005. Nucleosome and chromatin fiber dynamics. Curr Opin Struct Biol 15: 188-196.

Luger K, Mäder AW, Richmond RK, Sargent DF, Richmond TJ. 1997. Crystal structure of the nucleosome core particle at $2.8 \mathrm{~A}$ resolution. Nature 389: 251-260.

Maeshima K, Imai R, Hikima T, Joti Y. 2014. Chromatin structure revealed by X-ray scattering analysis and computational modeling. Methods 70: 154-161.
McCullough L, Connell Z, Petersen C, Formosa T. 2015. The Abundant histone chaperones Spt6 and FACT collaborate to assemble, inspect, and maintain chromatin structure in Saccharomyces cerevisiae. Genetics 201: 1031-1045.

McGinty RK, Tan S. 2015. Nucleosome structure and function. Chem Rev 115: 2255-2273.

Meng H, Andresen K, van Noort J. 2015. Quantitative analysis of single-molecule force spectroscopy on folded chromatin fibers. Nucleic Acids Res 43: 3578-3590.

Nishino Y, Eltsov M, Joti Y, Ito K, Takata H, Takahashi Y, Hihara S, Frangakis AS, Imamoto N, Ishikawa T, et al. 2012. Human mitotic chromosomes consist predominantly of irregularly folded nucleosome fibres without a $30-\mathrm{nm}$ chromatin structure. EMBO J 31: 1644-1653.

Orphanides G, LeRoy G, Chang C-H, Luse DS, Reinberg D. 1998. FACT, a factor that facilitates transcript elongation through nucleosomes. Cell 92: 105-116.

Orphanides G, Wu WH, Lane WS, Hampsey M, Reinberg D. 1999. The chromatin-specific transcription elongation factor FACT comprises human SPT16 and SSRP1 proteins. Nature 400: $284-288$.

Ou HD, Phan S, Deerinck TJ, Thor A, Ellisman MH, O'Shea CC. 2017. ChromEMT: Visualizing 3D chromatin structure and compaction in interphase and mitotic cells. Science 357: eaag0025.

Pope LH, Bennink ML, van Leijenhorst-Groener KA, Nikova D, Greve J, Marko JF. 2005. Single chromatin fiber stretching reveals physically distinct populations of disassembly events. Biophys $J$ 88: 3572-3583.

Risca VI, Denny SK, Straight AF, Greenleaf WJ. 2017. Variable chromatin structure revealed by in situ spatially correlated DNA cleavage mapping. Nature 541: 237-241.

Saunders A, Werner J, Andrulis ED, Nakayama T, Hirose S, Reinberg D, Lis JT. 2003. Tracking FACT and the RNA polymerase II elongation complex through chromatin in vivo. Science 301: 1094-1096.

Schalch T, Duda S, Sargent DF, Richmond TJ. 2005. X-ray structure of a tetranucleosome and its implications for the chromatin fibre. Nature 436: 138-141.

Scheffer MP, Eltsov M, Frangakis AS. 2011. Evidence for shortrange helical order in the $30-\mathrm{nm}$ chromatin fibers of erythrocyte nuclei. Proc Natl Acad Sci 108: 16992-16997.

Schwartz BE, Ahmad K. 2005. Transcriptional activation triggers deposition and removal of the histone variant H3.3. Genes Dev 19: 804-814.

Song F, Chen P, Sun D, Wang M, Dong L, Liang D, Xu RM, Zhu P, Li G. 2014. Cryo-EM study of the chromatin fiber reveals a double helix twisted by tetranucleosomal units. Science 344: 376-380.

Stuwe T, Hothorn M, Lejeune E, Rybin V, Bortfeld M, Scheffzek K, Ladurner AG. 2008. The FACT Spt16 "peptidase" domain is a histone H3-H4 binding module. Proc Natl Acad Sci 105: 8884-8889.

Syed SH, Goutte-Gattat D, Becker N, Meyer S, Shukla MS, Hayes JJ, Everaers R, Angelov D, Bednar J, Dimitrov S. 2010. Single-base resolution mapping of H1-nucleosome interactions and 3D organization of the nucleosome. Proc Natl Acad Sci 107: 9620-9625.

Tachiwana H, Kagawa W, Shiga T, Osakabe A, Miya Y, Saito K, Hayashi-Takanaka Y, Oda T, Sato M, Park SY, et al. 2011. Crystal structure of the human centromeric nucleosome containing CENP-A. Nature 476: 232-235.

Thakar A, Gupta P, Ishibashi T, Finn R, Silva-Moreno B, Uchiyama S, Fukui K, Tomschik M, Ausio J, Zlatanova J. 2009. H2A.Z and H3.3 histone variants affect nucleosome structure: Biochemical and biophysical studies. Biochemistry 48: $10852-10857$.

Thoma F, Koller T, Klug A. 1979. Involvement of histone H1 in the organization of the nucleosome and of the saltdependent superstructures of chromatin. J Cell Biol 83: 403427.

Thomas JO. 1999. Histone H1: Location and role. Curr Opin Cell Biol 11: 312-317. 
Tosi A, Haas C, Herzog F, Gilmozzi A, Berninghausen O, Ungewickell C, Gerhold CB, Lakomek K, Aebersold R, Beckmann R, et al. 2013. Structure and subunit topology of the INO80 chromatin remodeler and its nucleosome complex. Cell 154: $1207-1219$.

Tsunaka Y, Fujiwara Y, Oyama T, Hirose S, Morikawa K. 2016. Integrated molecular mechanism directing nucleosome reorganization by human FACT. Genes Dev 30: 673-686.

van der Heijden GW, Derijck AA, Pósfai E, Giele M, Pelczar P, Ramos L, Wansink DG, van der Vlag J, Peters AH, de Boer P. 2007. Chromosome-wide nucleosome replacement and H3.3 incorporation during mammalian meiotic sex chromosome inactivation. Nat Genet 39: 251-258.

van Holde K, Zlatanova J. 1996. Chromatin architectural proteins and transcription factors: A structural connection. Bioessays 18: $697-700$.

Watson JD, Crick FH. 1953. Molecular structure of nucleic acids; a structure for deoxyribose nucleic acid. Nature 171: 737-738.

Widom J, Klug A. 1985. Structure of the 300A chromatin filament: X-ray diffraction from oriented samples. Cell 43: 207-213.

Williams SP, Athey BD, Muglia LJ, Schappe RS, Gough AH Langmore JP. 1986. Chromatin fibers are left-handed double helices with diameter and mass per unit length that depend on linker length. Biophys $J$ 49: 233-248.

Wong LH, McGhie JD, Sim M, Anderson MA, Ahn S, Hannan RD, George AJ, Morgan KA, Mann JR, Choo KH. 2010. ATRX interacts with H3.3 in maintaining telomere structural integrity in pluripotent embryonic stem cells. Genome Res 20: 351-360.

Woodcock CL. 1994. Chromatin fibers observed in situ in frozen hydrated sections. Native fiber diameter is not correlated with nucleosome repeat length. J Cell Biol 125: 11-19.

Woodcock CL, Frado LL, Rattner JB. 1984. The higher-order structure of chromatin: Evidence for a helical ribbon arrangement. J Cell Biol 99: 42-52.

Yu Z, Zhou X, Wang W, Deng W, Fang J, Hu H, Wang Z, Li S, Cui L, Shen J, et al. 2015. Dynamic phosphorylation of CENP$A$ at Ser68 orchestrates its cell-cycle-dependent deposition at centromeres. Dev Cell 32: 68-81.

Zhang W, Zeng F, Liu Y, Shao C, Li S, Lv H, Shi Y, Niu L, Teng M, Li X. 2015. Crystal structure of human SSRP1 middle domain reveals a role in DNA binding. Sci Rep 5: 18688.

Zhu P, Li G. 2016. Structural insights of nucleosome and the 30nm chromatin fiber. Curr Opin Struct Biol 36: 106-115. 


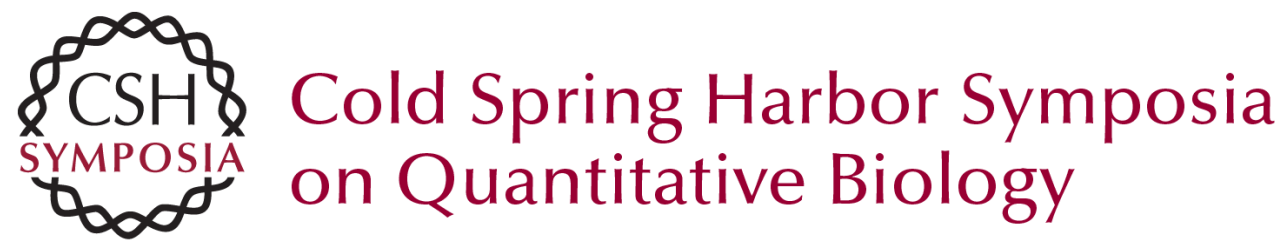

\section{Structure and Epigenetic Regulation of Chromatin Fibers}

Ping Chen and Guohong Li

Cold Spring Harb Symp Quant Biol 2017 82: 25-35 originally published online November 22, 2017

Access the most recent version at doi:10.1101/sqb.2017.82.033795

References This article cites 88 articles, 35 of which can be accessed free at: http://symposium.cshlp.org/content/82/25.full.html\#ref-list-1

Creative This article is distributed under the terms of the

Commons http://creativecommons.org/licenses/by-nc/4.0/, which permits reuse and License redistribution, except for commercial purposes, provided that the original author and source are credited.

Email Alerting Receive free email alerts when new articles cite this article - sign up in Service the box at the top right corner of the article or click here. 